\title{
33. INTERSTITIAL WATER STUDIES ON SMALL CORE SAMPLES, LEG $19^{1}$
}

\author{
Frederick L. Sayles and Lee S. Waterman, Woods Hole Oceanographic Institution, Woods Hole, Massachusetts \\ and \\ Frank T. Manheim, United States Geological Survey, Woods Hole, Massachusetts
}

The sediments cored on Leg 19 consist primarily of diatomaceous oozes with variable proportions of volcanic material and terrigenous clays and silts. With a few exceptions, deposition rates are high at these sites, usually exceeding $5 \mathrm{~cm} / 10^{3} \mathrm{y}$. The interstitial solutions sampled exhibit compositional changes which previously have been found to characterize rapidly deposited tertigenous sediments. Some of the largest changes in $\mathrm{Na}^{+}, \mathrm{K}^{+}, \mathrm{Mg}^{2+}$, $\mathrm{Cl}^{-}$, and $\mathrm{HCO}_{3}$ yet found in DSDP samples occur in the pore waters studied from this leg (see Table 1).

Depletion of interstitial $\mathrm{SO}_{4}{ }^{2-}$ is extensive at many of the Leg 19 sites $(185,186,189$, and 191), and alkalinities of more than ten times that of ocean water are associated with these $\mathrm{SO}_{4}{ }^{2-}$ depletions. Although some of the $\mathrm{CA}^{2+}$ loss observed at the latter sites may be an artifact caused by post-sampling precipitation of $\mathrm{CaCO}_{3}$ (Gieskes, in press), real depletion of $\mathrm{Ca}^{2+}$ in the pore solutions is inferred to occur. In accord with earlier observations, alkalinities often decrease towards the bottom of the holes. This is most markedly demonstrated at Sites 185,186 , and 189 where alkalinities in the deepest samples are as much as $15 \mathrm{meq} / \mathrm{kg}$ lower than in the uppermost ones. $\mathrm{Ca}^{2+}$, which has been depleted in the higher portions of the sedimentary section, begins to increase at depths of 100 to 200 meters as the alkalinity falls. Eventually, $\mathrm{Ca}^{2+}$ becomes enriched relative to ocean water at Sites 185, 186, 189, and 192. Large depletions of $\mathrm{Mg}^{2+}$ occur at all of the Leg 19 sites. The $\mathrm{Mg}^{2+}$ concentrations observed at Sites 184,185 , and 186 are the lowest yet found in DSDP samples $(<0.2 \mathrm{gm} / \mathrm{kg})$.

The concentrations of both $\mathrm{Na}^{+}$and $\mathrm{Cl}^{-}$are significantly lower than those of mean ocean water in the deeper samples from Sites 184, 185, 186, and 189. At Site 186 , the chlorinity at 108 meters is almost $20 \%$ less than that of mean ocean water. Similar decreases of $\mathrm{Na}^{+}$and $\mathrm{Cl}^{-}$ have been observed in samples from Legs 11 and 15.

The observed decrease in $\mathrm{Cl}^{-}\left(\right.$and $\left.\mathrm{Na}^{+}\right)$concentrations must be due to dilution because $\mathrm{Cl}^{-}$cannot be taken up appreciably by the solid phases of the sediment. The source of the fresh solutions is uncertain. All of the sites exhibiting such $\mathrm{Cl}^{-}$decreases are characterized by clay-rich sediments and rapid sedimentation. Artesian flow such as found 120 $\mathrm{km}$ off the coast of Florida (Manheim, 1967) seems unlikely but is a possibility. The sites at which low $\mathrm{Cl}^{-}$ values occur are adjacent to the Aleutian Island chain and may be underlain by older sedimentary basement. Artesian flow could occur in the basement rock and cannot be ruled out without more extensive structural information. Chemical reactions (e.g., oxidation of organic matter) can

\footnotetext{
${ }^{1}$ Contribution Number 2461 of the Woods Hole Oceanographic Institution.
}

produce $\mathrm{H}_{2} \mathrm{O}$ in sediments, but the quantities produced in normal marine sediments are trivial compared to the amounts required. The fresh water could be supplied by the clays themselves, being released from the interlayers during burial and compaction. However, the complete removal of interlayer water from montmorillonite requires temperatures approaching $100^{\circ} \mathrm{C}$ (Grim, 1968). Such temperatures cannot be found at the depths where $\mathrm{Cl}^{-}$depletion is found, especially at Site 186 . The source of the considerable quantities of fresh water required thus represents an unsolved and intriguing question.

The $\mathrm{Na} / \mathrm{Cl}$ ratio also deviates from the value characteristic of ocean water $(0.556)$. The ratio for most of the samples (except at Sites 183, 188, and 191) is significantly higher than that of ocean water, falling in the range 0.560 to 0.575 . In contrast, pelagic sediments are characterized by ratios which scatter about the ocean water value, seldom deviating by more than \pm 0.007 . The observed departures from the ocean water value are close to our detection limit, but are significant. The estimated accuracy in the determination of $\mathrm{Na} / \mathrm{Cl}$ is \pm 0.007 ; a Copenhagen seawater standard treated exactly as the samples yielded a $\mathrm{Na} / \mathrm{Cl}$ ratio of 0.552 .

The increases in $\mathrm{Na} / \mathrm{Cl}$ found at most of the sites on this leg are the best documented evidence to date of the reaction of $\mathrm{Na}^{+}$in the pore fluids. $\mathrm{As} \mathrm{Cl}^{-}$does not react appreciably with the solid phases of the sediment, the increase in the $\mathrm{Na} / \mathrm{Cl}$ ratio must then be indicative of the release of $\mathrm{Na}^{+}$into solution by the enclosing solids. A similar increase in $\mathrm{Na} / \mathrm{Cl}$ was also found at several of the Leg 11 sites $\left(102,104\right.$, and 106). Such an increase in $\mathrm{Na}^{+}$ relative to $\mathrm{Cl}^{-}$might be anticipated; the sediments at sites exhibiting this effect contain appreciable amounts of unweathered terrigenous detritus such as feldspars. Weathering of both plagioclase and alkali feldspar will release $\mathrm{Na}^{+}$to solution. We should note, however, that exceptions to this increase in $\mathrm{Na}^{+}$do exist. Significant depletion of $\mathrm{Na}^{+}$relative to $\mathrm{Cl}^{-}$is indicated by a decrease in $\mathrm{Na} / \mathrm{Cl}$ below the ocean water value at Sites 105 (Leg 11) and 148 (Leg 15). No precise knowledge of the reactions involved are available.

\section{REFERENCES}

Gieskes, J. M., in press. Interstitial water studies, Leg 15. In Edgar, N. T., Saunders, J. B. et al. Initial Reports of the Deep Sea Drilling Project, Volume XV.

Grim, R. E., 1968. Clay Mineralogy. New York (McGraw-Hill) [2nd Ed.]. Ch. 8.

Manheim, F. T., 1967. Evidence for submarine discharge of water on the Atlantic continental slope of the southern United States, and suggestions for further search. Trans. New York Acad. Sciences, Ser. II. 29, 839. 
TABLE 1

Major Constituents and Si Content of Pore Fluids

\begin{tabular}{|c|c|c|c|c|c|c|c|c|c|c|c|c|c|c|c|c|c|c|c|}
\hline Sample & $\begin{array}{l}\text { Depth } \\
\text { (m) }\end{array}$ & Age & Description & $\underset{(\mathrm{g} / \mathrm{kg})}{\mathrm{Na}^{\mathrm{a}}}$ & $\underset{(\mathrm{g} / \mathrm{kg})}{\mathrm{Na}^{\mathrm{b}}}$ & $\underset{(\mathrm{g} / \mathrm{kg})}{\mathrm{K}}$ & $\begin{array}{c}\mathrm{Ca} \\
(\mathrm{g} / \mathrm{kg})\end{array}$ & $\begin{array}{c}\mathrm{Mg} \\
(\mathrm{g} / \mathrm{kg})\end{array}$ & $\begin{array}{l}\text { Total } \\
\text { Cations } \\
\text { (meq/kg) }\end{array}$ & $\underset{(\mathrm{g} / \mathrm{kg})}{\mathrm{Cl}}$ & $\begin{array}{c}\mathrm{SO}_{4} \\
(\mathrm{~g} / \mathrm{kg})\end{array}$ & $\underset{(\mathrm{meq} / \mathrm{kg})}{\text { Alk. }}$ & $\begin{array}{l}\mathrm{HCO}_{3} \mathrm{c} \\
(\mathrm{g} / \mathrm{kg})\end{array}$ & $\begin{array}{c}\text { Total } \\
\text { Anions } \\
(\mathrm{meq} / \mathrm{kg})\end{array}$ & $\begin{array}{l}\text { Sumd } \\
(\mathrm{g} / \mathrm{kg})\end{array}$ & Salinity $^{\mathrm{e}}$ & $\stackrel{\mathrm{O}_{2} \mathrm{O}}{\mathrm{H}}$ & $\mathrm{pH}^{\mathrm{f}}$ & $\underset{(\mathrm{ppm})}{\mathrm{Si}}$ \\
\hline \multicolumn{20}{|c|}{ Site $183\left(52^{\circ} 34.3^{\prime} \mathrm{N}, 161^{\circ} 12.3^{\prime} \mathrm{W}\right.$, water depth $4,708 \mathrm{~m}$, Aleutian Abyssal Plain) } \\
\hline $183-11-5$ & 106 & Pliocene & $\begin{array}{l}\text { Olive gray silty and } \\
\text { silt-rich clays; large } \\
\text { proportions of } \\
\text { diatoms. }\end{array}$ & 10.9 & 10.9 & 0.34 & 0.57 & 1.11 & 601 & 19.4 & 2.23 & 8.9 & 0.54 & 602 & 35.1 & 34.7 & 37 & 8.1 & 16.8 \\
\hline $28-3$ & 270 & Oligocene (?) & Olive gray silt. & 10.9 & 10.9 & 0.18 & 0.60 & 1.20 & 607 & 19.4 & 2.53 & 5.7 & 0.34 & 607 & 35.2 & 35.2 & - & 7.9 & 3.3 \\
\hline \multicolumn{20}{|c|}{ Site $184\left(53^{\circ} 42.6^{\prime} \mathrm{N}, 170^{\circ} 55.4^{\prime} \mathrm{W}\right.$, water depth $1,910 \mathrm{~m}$, Umnak Plateau) } \\
\hline $184-2-6$ & 136 & Pliocene & $\begin{array}{l}\text { Olive gray diatom } \\
\text { ooze. }\end{array}$ & 11.2 & 11.0 & 0.34 & 0.53 & 0.87 & 586 & 19.4 & 1.87 & 8.0 & 0.49 & 593 & 34.7 & 33.8 & - & 7.6 & 25 \\
\hline $4-4$ & 170 & Pliocene & $\begin{array}{l}\text { Grayish olive diatom } \\
\text { ooze; bedding moder- } \\
\text { ately deformed. }\end{array}$ & 11.2 & 11.0 & 0.33 & 0.59 & 0.85 & 589 & 19.4 & 1.91 & 7.2 & 0.44 & 594 & 34.7 & 33.8 & 55 & 7.6 & 26 \\
\hline $7-6$ & 228 & Pliocene & $\begin{array}{l}\text { Dark olive gray diatom } \\
\text { ooze; bedding moder- } \\
\text { ately deformed. }\end{array}$ & 11.1 & 11.0 & 0.33 & 0.68 & 0.85 & 591 & 19.4 & 1.95 & 3.6 & 0.22 & 592 & 34.5 & 34.1 & - & 7.7 & 26 \\
\hline $10-4$ & 282 & Pliocene & $\begin{array}{l}\text { Dark olive gray clay- } \\
\text { rich diatom ooze; } \\
\text { bedding moderately } \\
\text { deformed. }\end{array}$ & & 11.0 & 0.33 & 0.75 & 0.81 & 590 & 19.4 & 1.99 & - & - & - & - & 33.8 & - & 7.7 & 29 \\
\hline $14-3$ & 383 & Upper Miocene & $\begin{array}{l}\text { Dark olive gray clay- } \\
\text { rich diatom ooze; } \\
\text { bedding moderately } \\
\text { deformed. }\end{array}$ & 11.0 & 10.9 & 0.33 & 0.89 & 0.70 & 586 & 19.2 & 1.92 & 6.9 & 0.42 & 589 & 34.5 & 33.6 & - & 8.0 & 25 \\
\hline $20-5$ & 545 & Upper Miocene & $\begin{array}{l}\text { Dark olive gray clay- } \\
\text { rich diatom ooze; } \\
\text { bedding moderately } \\
\text { deformed. }\end{array}$ & 10.6 & 10.7 & 0.24 & 1.51 & 0.31 & 573 & 19.0 & 1.34 & 6.2 & 0.38 & 569 & 33.4 & 33.0 & - & 7.6 & 31 \\
\hline $22-6$ & 598 & Upper Miocene & $\begin{array}{l}\text { Dark olive gray } \\
\text { clayey diatom ooze. }\end{array}$ & - & 10.6 & 0.20 & $(1.7)^{\mathrm{g}}$ & 0.19 & - & 18.9 & 1.16 & 3.4 & 0.21 & 562 & - & 32.2 & - & - & 24 \\
\hline \multicolumn{20}{|c|}{ Site $185\left(54^{\circ} 25.7^{\prime} \mathrm{N}, 169^{\circ} 14.6^{\prime} \mathrm{W}\right.$, water depth $2,110 \mathrm{~m}$, Umnak Plateau) } \\
\hline $185-4-6$ & 42 & Pleistocene & $\begin{array}{l}\text { Dark grayish olive to } \\
\text { grayish olive diatom } \\
\text { silty clay. }\end{array}$ & 11.0 & 10.8 & 0.41 & 0.09 & 1.01 & 569 & 19.5 & 0.29 & 22.2 & 1.35 & 577 & 33.6 & 33.0 & 46 & 7.8 & 24 \\
\hline $6-6$ & 98 & Pleistocene & $\begin{array}{l}\text { Various shades of } \\
\text { olive gray silty clay- } \\
\text { rich diatom ooze. }\end{array}$ & 11.2 & 10.9 & 0.38 & 0.06 & 0.81 & 552 & $19.3<$ & $<0.01$ & 20.1 & 1.23 & 564 & 33.0 & 32.5 & 46 & 8.0 & 22 \\
\hline $7-5$ & 135 & Pliocene & $\begin{array}{l}\text { Dark olive gray to } \\
\text { grayish olive silt and } \\
\text { clay-rich diatom ooze. }\end{array}$ & & 10.8 & 0.34 & 0.09 & 0.80 & 550 & $19.2<$ & $<0.01$ & - & - & - & - & 32.2 & - & 7.8 & 22 \\
\hline
\end{tabular}




\begin{tabular}{|c|c|c|c|c|c|c|c|c|c|c|c|c|c|c|c|c|c|c|c|}
\hline Sample & $\begin{array}{l}\text { Depth } \\
(\mathrm{m})\end{array}$ & Age & Description & $\underset{(\mathrm{g} / \mathrm{kg})}{\mathrm{Na}^{\mathrm{a}}}$ & $\begin{array}{r}\mathrm{Na}^{\mathrm{b}} \\
(\mathrm{g} / \mathrm{kg})\end{array}$ & $\underset{(\mathrm{g} / \mathrm{kg})}{\mathrm{K}}$ & $\begin{array}{c}\mathrm{Ca} \\
(\mathrm{g} / \mathrm{kg})\end{array}$ & $\underset{(\mathrm{g} / \mathrm{kg})}{\mathrm{Mg}}$ & $\begin{array}{c}\text { Total } \\
\text { Cations } \\
(\mathrm{meq} / \mathrm{kg})\end{array}$ & $\underset{(\mathrm{g} / \mathrm{kg})}{\mathrm{Cl}}$ & $\begin{array}{l}\mathrm{SO}_{4} \\
(\mathrm{~g} / \mathrm{kg})\end{array}$ & $\begin{array}{c}\text { Alk. } \\
\text { (meq/kg) }\end{array}$ & $\begin{array}{l}\mathrm{HCO}_{3}{ }^{\mathrm{C}} \\
(\mathrm{g} / \mathrm{kg})\end{array}$ & $\begin{array}{c}\text { Total } \\
\text { Anions } \\
\text { (meq/kg) }\end{array}$ & 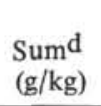 & Salinity ${ }^{\mathrm{e}}$ & $\left(\stackrel{0}{\mathrm{H}_{2} \mathrm{O}}\right.$ & $\mathrm{pH}^{\mathrm{f}}$ & $\begin{array}{c}\mathrm{Si} \\
\text { (ppm) }\end{array}$ \\
\hline $185-8-4$ & 172 & $\begin{array}{l}\text { Pliocene-Upper } \\
\text { Miocene }\end{array}$ & $\begin{array}{l}\text { Dark olive gray to } \\
\text { grayish olive clayey to } \\
\text { silty diatom ooze. }\end{array}$ & 11.1 & 10.9 & 0.33 & 0.08 & 0.77 & 549 & 19.3 & $<0.01$ & 16.2 & 0.99 & 560 & 32.6 & 31.9 & - & 7.8 & 27 \\
\hline $20-5$ & 668 & Upper Miocene & $\begin{array}{l}\text { Olive gray diatom- } \\
\text { rich silty clay. }\end{array}$ & 10.2 & 10.4 & 0.14 & 1.40 & 0.15 & 539 & 18.7 & $<0.01$ & 3.5 & 0.22 & 533 & 30.8 & 30.6 & - & 8.0 & 17.0 \\
\hline \multicolumn{20}{|c|}{ Site $186\left(51^{\circ} 07.8^{\prime} \mathrm{N}, 174^{\circ} 00.3^{\prime} \mathrm{W}\right.$, water depth $4,522 \mathrm{~m}$, north wall of Aleutian Trench) } \\
\hline $186-3-6$ & 18 & $\begin{array}{l}\text { Upper } \\
\text { Pleistocene }\end{array}$ & $\begin{array}{l}\text { Dark greenish gray } \\
\text { diatom ooze; gassy; } \\
\text { disturbed. }\end{array}$ & - & 10.1 & 0.38 & 0.07 & 0.84 & 521 & 17.8 & - & 34.7 & 2.12 & - & - & 30.8 & - & 8.1 & 22 \\
\hline $6-3$ & 108 & $\begin{array}{l}\text { Middle } \\
\text { Pleistocene }\end{array}$ & $\begin{array}{l}\text { Olive gray diatom } \\
\text { silty clay; gassy; } \\
\text { disturbed. }\end{array}$ & 9.4 & 9.1 & 0.33 & 0.10 & 0.72 & 470 & 15.7 & $<0.01$ & 39.4 & 2.40 & 482 & 26.7 & 27.5 & - & 8.2 & 22 \\
\hline $9-6$ & 168 & $\begin{array}{l}\text { Middle } \\
\text { Pleistocene }\end{array}$ & $\begin{array}{l}\text { Very dark gray diatom } \\
\text { silty clay; gassy; } \\
\text { disturbed. }\end{array}$ & 9.2 & 9.3 & 0.31 & 0.09 & 0.73 & 476 & 16.0 & $<0.01$ & 22.3 & 1.36 & 473 & 27.7 & 27.8 & - & 8.0 & 22 \\
\hline $20-4$ & 462 & Upper Miocene & $\begin{array}{l}\text { Olive gray, spicule } \\
\text { bearing diatom silty } \\
\text { clay. }\end{array}$ & 10.2 & 9.9 & 0.28 & 0.19 & 0.68 & 504 & 17.3 & 0.36 & 21.5 & 1.31 & 516 & 30.3 & 29.4 & - & 8.0 & 20 \\
\hline
\end{tabular}

\section{Site $188\left(53^{\circ} 45.2^{\prime} \mathrm{N}, 178^{\circ} 39.6^{\prime} \mathrm{E}\right.$, water depth $2,649 \mathrm{~m}$, inner flank of Bowers Ridge}

\begin{tabular}{|c|c|c|c|c|c|c|c|c|c|c|c|c|c|c|c|c|c|c|c|}
\hline $188-3-5$ & 33 & $\begin{array}{l}\text { Middle } \\
\text { Pleistocene }\end{array}$ & $\begin{array}{l}\text { Light olive gray } \\
\text { clayey silt rich } \\
\text { diatom ooze. }\end{array}$ & 11.0 & 10.9 & 0.42 & 0.40 & 1.24 & 605 & 19.5 & 2.43 & 10.7 & 0.65 & 612 & 35.6 & 35.2 & 48 & 7.9 & 20 \\
\hline $6-1$ & 125 & $\begin{array}{l}\text { Lower } \\
\text { Pleistocene }\end{array}$ & $\begin{array}{l}\text { Grayish olive clay- } \\
\text { rich diatom silt. }\end{array}$ & 11.0 & 10.8 & 0.42 & 0.29 & 1.20 & 594 & 19.5 & 2.00 & 10.9 & 0.66 & 604 & 35.1 & 35.2 & 50 & 8.0 & 26 \\
\hline $9-3$ & 230 & Pliocene & $\begin{array}{l}\text { Olive gray diatom } \\
\text { ooze. }\end{array}$ & 11.1 & 10.8 & 0.36 & 0.20 & 1.13 & 583 & 19.5 & 1.81 & 5.5 & 0.34 & 594 & 34.4 & 34.1 & - & 7.5 & 38 \\
\hline
\end{tabular}

Site $189\left(54^{\circ} 02.1^{\prime} \mathrm{N}, 170^{\circ} 13.4^{\prime} \mathrm{E}\right.$, water depth $3,437 \mathrm{~m}$, northern flank of Aleutian-Komandorsky Ridge)

\begin{tabular}{|c|c|c|c|c|c|c|c|c|c|c|c|c|c|c|c|c|c|c|}
\hline $189-4-3$ & 86 & $\begin{array}{l}\text { Lower } \\
\text { Pleistocene }\end{array}$ & $\begin{array}{l}\text { Dark greenish gray } \\
\text { diatom bearing silty } \\
\text { clay. }\end{array}$ & 10.8 & 10.8 & 0.39 & 0.20 & 0.90 & 565 & $19.2<0.01$ & 20.7 & 1.26 & 563 & 32.8 & 33.0 & - & 8.2 & 19.8 \\
\hline $7-2$ & 298 & Pliocene & $\begin{array}{l}\text { Olive gray silt-rich } \\
\text { diatom-carbonate- } \\
\text { pyrite bearing clay. }\end{array}$ & 10.9 & 10.8 & 0.26 & 0.48 & 0.44 & 537 & 0.24 & 5.4 & 0.33 & 543 & 31.6 & 31.1 & - & 8.2 & 15.0 \\
\hline
\end{tabular}

Site $190\left(55^{\circ} 33.6^{\prime} \mathrm{N}, 171^{\circ} 38.4^{\prime} \mathrm{E}\right.$, water depth $3,875 \mathrm{~m}$, Aleutian Basin)

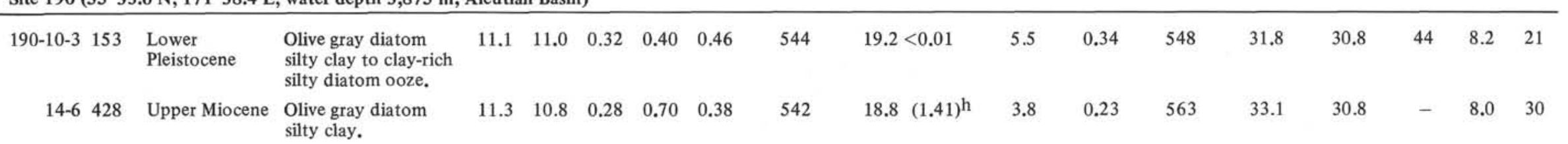


TABLE 1 - Continued

\begin{tabular}{|c|c|c|c|c|c|c|c|c|c|c|c|c|c|c|c|c|c|c|c|}
\hline Sample & $\begin{array}{l}\text { Depth } \\
\text { (m) }\end{array}$ & Age & Description & $\begin{array}{c}\mathrm{Na}^{\mathrm{a}} \\
(\mathrm{g} / \mathrm{kg})\end{array}$ & $\begin{array}{r}\mathrm{Na}^{\mathrm{b}} \\
(\mathrm{g} / \mathrm{kg})\end{array}$ & $\underset{(\mathrm{g} / \mathrm{kg})}{\mathrm{K}}$ & $\begin{array}{c}\mathrm{Ca} \\
(\mathrm{g} / \mathrm{kg})\end{array}$ & $\begin{array}{c}\mathrm{Mg} \\
(\mathrm{g} / \mathrm{kg})\end{array}$ & $\begin{array}{c}\text { Total } \\
\text { Cations } \\
(\mathrm{meq} / \mathrm{kg})\end{array}$ & $\begin{array}{c}\mathrm{Cl} \\
(\mathrm{g} / \mathrm{kg})\end{array}$ & $\begin{array}{l}\mathrm{SO}_{4} \\
(\mathrm{~g} / \mathrm{kg})\end{array}$ & $\begin{array}{c}\text { Alk. } \\
\text { (meq } / \mathrm{kg})\end{array}$ & $\underset{(\mathrm{g} / \mathrm{kg})}{\mathrm{HCO}_{3}{ }^{\mathrm{c}}}$ & $\begin{array}{c}\text { Total } \\
\text { Anions } \\
\text { (meq } / \mathrm{kg} \text { ) }\end{array}$ & $\begin{array}{l}\text { Sum }^{\mathrm{d}} \\
(\mathrm{g} / \mathrm{kg})\end{array}$ & Salinity ${ }^{e}$ & ${ }_{(\%}^{\circ} \mathrm{O}_{2} \mathrm{H}^{\mathrm{O}} \mathrm{O}$ & $\mathrm{pH}^{\mathrm{f}}$ & $\begin{array}{c}\mathrm{Si} \\
(\mathrm{ppm})\end{array}$ \\
\hline \multicolumn{20}{|c|}{ Site-191 ( $56^{\circ} 56.7^{\prime} \mathrm{N}, 168^{\circ} 10.7^{\prime} \mathrm{E}$, water depth $3,854 \mathrm{~m}$, Kamchatka Basin) } \\
\hline $191-4-6$ & 86 & $\begin{array}{l}\text { Middle } \\
\text { Pleistocene }\end{array}$ & $\begin{array}{l}\text { Olive gray silt-rich } \\
\text { clayey diatom ooze. }\end{array}$ & 10.7 & 10.8 & 0.36 & 0.18 & 1.11 & 580 & 19.5 & $<0.01$ & 26.7 & 1.63 & 576 & 33.5 & 34.4 & 47 & 8.0 & 19.5 \\
\hline $5-6$ & 142 & $\begin{array}{l}\text { Middle } \\
\text { Pleistocene }\end{array}$ & $\begin{array}{l}\text { Very dark gray diatom- } \\
\text { rich silty clay. }\end{array}$ & 11.2 & 10.9 & 0.33 & 0.10 & 0.96 & 567 & 19.5 & $<0.01$ & 30.2 & 1.84 & 580 & 33.9 & 33.8 & 36 & 8.3 & 15.0 \\
\hline \multicolumn{20}{|c|}{ Site $192\left(53^{\circ} 00.6^{\prime} \mathrm{N}, 164^{\circ} 42.8^{\prime} \mathrm{E}\right.$, water depth $3,014 \mathrm{~m}$, Emperor Seamount Chain) } \\
\hline $192-4-6$ & 26 & Pleistocene & $\begin{array}{l}\text { Olive gray diatoma- } \\
\text { ceous silty clay. }\end{array}$ & 10.5 & 10.9 & 0.45 & 0.35 & 1.11 & 592 & 19.5 & 0.87 & 10.0 & 0.61 & 578 & 33.4 & 34.4 & 45 & 8.5 & 17.0 \\
\hline $7-4$ & 78 & Pliocene & $\begin{array}{l}\text { Dark greenish gray } \\
\text { diatomaceous silty } \\
\text { clay. }\end{array}$ & 10.8 & 10.9 & 0.40 & 0.27 & 0.95 & 575 & 19.5 & 0.70 & 7.7 & 0.47 & 572 & 33.1 & 33.3 & 43 & 8.2 & 18.5 \\
\hline $9-6$ & 128 & Pliocene & $\begin{array}{l}\text { Dark greenish gray } \\
\text { diatomaceous silty } \\
\text { clay. }\end{array}$ & 10.9 & 11.0 & 0.41 & 0.25 & 0.83 & 570 & 19.6 & 0.30 & 9.2 & 0.56 & 567 & 32.8 & 33.0 & 42 & 8.4 & 17.5 \\
\hline $15-4$ & 274 & $\begin{array}{l}\text { Upper } \\
\text { Miocene }\end{array}$ & $\begin{array}{l}\text { Olive gray clay-rich } \\
\text { diatom ooze. }\end{array}$ & 11.2 & 11.1 & 0.38 & 0.28 & 0.69 & 563 & 19.6 & 0.30 & 6.2 & 0.38 & 567 & 32.8 & 32.8 & 50 & 8.1 & 26 \\
\hline $17-3$ & 328 & $\begin{array}{l}\text { Upper } \\
\text { Miocene }\end{array}$ & $\begin{array}{l}\text { Dark gray clay-rich } \\
\text { diatom ooze. }\end{array}$ & 11.2 & 11.2 & 0.38 & 0.33 & 0.65 & 569 & 19.6 & 0.37 & 5.3 & 0.32 & 567 & 32.8 & 32.5 & - & 8.2 & 25 \\
\hline
\end{tabular}

aSodium determined by difference between anions and cations excluding $\mathrm{Na}$.

bSodium determined by atomic absorption analysis.

$\mathrm{CHCO}_{3}$ is calculated from total alkalinity, assuming this is entirely due to bicarbonate ion.

$\mathrm{d}$ The sum incorporates the sodium values determined by difference.

eSalinity of pore fluids taken from heat-sealed sections of plastic pipe prior to subdivision of samples for analysis. Salinity values determined with Goldberg temperature-compensated refractometer.

Water content and $\mathrm{pH}$ are taken from shipboard summaries.

gCalcium determined by difference for Sample 184-22-6.

$\mathrm{h}_{\mathrm{The}} \mathrm{SO}_{4}$ of this sample appears to be in error, as it is considerably higher than would be expected. Such an error would account for the poor agreement between the Na by difference and analysis, and also between the sum and the salinity. 